\section{Incidence of upper-extremity deep vein thrombosis in western France: a community-based study}

Proximal upper-extremity deep vein thrombosis (UEDVT) is defined by thrombosis of the brachial, axillary, or subclavian veins. It also includes extension to the brachiocephalic vein, superior vena cava, and internal jugular vein. ${ }^{1}$ UEDVT is usually classified into primary and secondary forms. Primary UEDVT encompasses unprovoked thrombosis and thrombosis associated with thoracic outlet syndrome. Secondary UEDVT is associated with a known risk factor, which is most frequently the presence of a central venous catheter. ${ }^{2}$ Unlike lowerextremity deep vein thrombosis, the association between UEDVT and symptomatic pulmonary embolism is not common. The frequency of symptomatic pulmonary embolism associated with a symptomatic UEDVT is not known precisely but is estimated to be between $3 \%$ and $12 \%{ }^{3}$

To date, estimates of the incidence of UEDVT have only been obtained through the extrapolation of the proportion of UEDVT recorded in registries of highly selected patients with venous thromboembolism. ${ }^{3-6}$ UEDVT may account for $10 \%$ of the number of deep vein thromboses of the extremities; however, the current incidence of symptomatic UEDVT may be higher than expected because of the dramatic increase in the use of central venous catheters over the last years.

The EPIGETBO study is an epidemiological study that prospectively enrolled all patients living in a well-defined geographic area in western France who were diagnosed in hospitals or in the community with symptomatic venous thromboembolism. ${ }^{7}$ The purpose of this analysis of the EPIGETBO data was to determine the incidence of and risk factors for symptomatic UEDVT occurring over a 2-year period.

The methods of the EPIGETBO study have already been extensively described. ${ }^{7}$ All patients aged 18 years or older living in the Brest District between March $1^{\text {st }}, 2013$ and February $28^{\text {th }}, 2015$ with an objectively documented, symptomatic, proximal deep vein thrombosis of the extremities were prospectively included in the study. Symptomatic UEDVT was defined as the presence of swelling, pain, and/or redness of the upper extremity. UEDVT had to be confirmed by compression ultrasonography or a computed tomography angiogram. UEDVT patients with no symptoms suggestive of pulmonary embolism did not undergo systematic chest imaging.

A standardized case report form was filled in for every patient with proven acute venous thromboembolism. All patients or their families were interviewed directly or by telephone in order to collect clinical data, and the charts of admitted patients were reviewed. The patients' general demographic characteristics, risk factors for venous thromboembolism, clinical signs and symptoms, date and location of venous thromboembolism and place of diagnosis of venous thromboembolism were recorded. Patients were contacted at least twice to ensure the accuracy of the recorded data and modifications in health condition.

The database was checked regularly in order to exclude double entries and to review, validate, and adjudicate the diagnoses of venous thromboembolism. Proximal UEDVT was defined as thrombosis of the brachial vein or a more proximal vein. Etiological workup was left at the discretion of physicians in charge of the patients.

Clinical characteristics are described by the mean \pm standard derivation and by numbers and percentages.
Annual incidence rates of UEDVT per 1,000 inhabitants were calculated as the number of UEDVT cases occurring between March $1^{\text {st }}, 2013$ and February $28^{\text {th }}, 2015$, divided by two and by the population of the Brest District, which was 367,911 as per the 2013 census performed by the National Institute for Statistics and Economics Studies (INSEE). ${ }^{8}$ The $95 \%$ confidence intervals (CI) were estimated based on a Poisson distribution.

The study protocol was approved by the Brest University Hospital Ethics Committee. Individual written consent was not required.

Between March $1^{\text {st }}, 2013$ and February 28 ${ }^{\text {th }}, 2015,502$ inhabitants of the Brest District were diagnosed with a symptomatic proximal deep vein thrombosis of the extremities. Of them, $72(14.3 \%)$ had a symptomatic proximal UEDVT. The mean age of UEDVT patients was $63.2 \pm 18.6$ years, $33(45.8 \%)$ were men, $48(66.75)$ had a central venous catheter, and active cancer was also reported in $48(66.7 \%)$ (Table 1$)$. The central venous catheters were peripherally inserted central catheters $(n=20)$, port-a-cath $(n=17)$ ports, double or triple lumen catheters $(n=10)$, or not specified $(n=1)$. Of the 48 patients with cancer, $34(70.8 \%)$ had had a central venous catheter placed shortly before or at the time of diagnosis of UEDVT. The UEDVT was more proximal in patients with cancer than in those without cancer: $39 / 48$ $(81.25 \%)$ cases of UEDVT in cancer patients extended to the subclavian vein or internal jugular vein whereas $11 / 24(45.8 \%)$ cases did so in non-cancer patients $(P=0.003)$. A symptomatic pulmonary embolism was diagnosed concurrently in six of the 72 patients with UEDVT $(8.3 \%)$. Five out of these six patients $(83.3 \%)$ had a central venous catheter in place. The proportion of patients with pulmonary embolism was not different between patients with primary and secondary UEDVT $(P=0.47)$.

The overall annual incidence rate of UEDVT was 0.098 per 1,000 inhabitants (95\% CI: 0.097 to 0.099). The incidence of UEDVT increased with increasing age (Figure 1A): the annual incidence was 0.047 per 1,000 (95\% CI:

Table 1. Clinical characteristics of the 72 patients with symptomatic upper-extremity deep vein thrombosis.

\begin{tabular}{lc} 
Characteristic & $N=72$ \\
Age, mean (SD) & $63.2 \pm 18.6$ \\
Men & $33(45.9 \%)$ \\
\hline Smokers & $37(55.2 \%)$ \\
Past history of VTE & $11(15.9 \%)$ \\
\hline Family history of VTE & $6(10.7 \%)$ \\
Admission to hospital within 3 months & $35(49.3 \%)$ \\
(medical wards) & \\
\hline Active cancer & $48(66.7 \%)$ \\
Surgery within 3 months & $19(26.8 \%)$ \\
\hline Pregnancy/post-partum & $1(2.6 \%)$ \\
Oral contraceptives & $2(5.3 \%)$ \\
\hline Hormone replacement therapy & $1(2.6 \%)$ \\
Central venous catheter & $48(66.7 \%)$ \\
Associated with cancer & $34(70.8 \%)^{*}$ \\
Not associated with cancer & $14(29.2 \%)^{*}$ \\
\hline Symptomatic pulmonary embolism & $6(8.3 \%)$
\end{tabular}

Symptomatic pulmonary embolism $6(8.3 \%)$

VTE: venous thromboembolism. *among patients with central venous catheter in place 
A

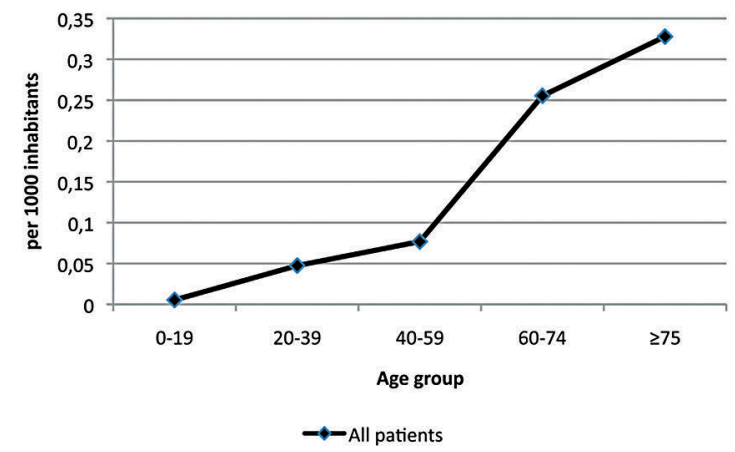

B

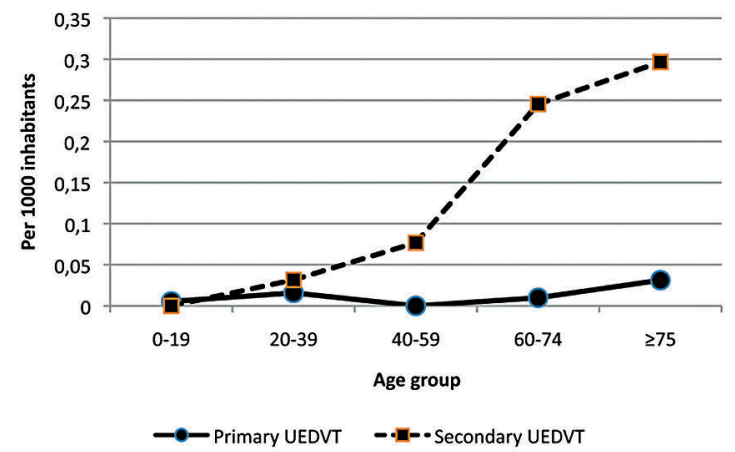

Figure 1. Annual incidence of symptomatic upper-extremity deep vein thrombosis UEDVT: upper extremity deep vein thrombosis.

0.046 to 0.049 ) in residents aged $20-39$ years and reached 0.328 per 1,000 (95\% CI: 0.321 to 0.334$)$ in residents aged 75 years and over. No difference in incidence rates was observed between men and women.

Primary UEDVT occurred in seven patients $(9.7 \%)$. The annual incidence of primary UEDVT was 0.0095 per 1,000 (95\% CI: 0.0092 to 0.0098$)$ and was stable across age categories (Figure 1B). In contrast, the annual incidence of secondary UEDVT was higher in older patients than in younger ones: 0.297 per 1,000 (95\% CI: 0.291 to 0.303 ) in patients 75 years and over versus 0.032 per 1,000 (95\% CI: $0.031-0.033$ ) in patients aged $20-39$ years (incidence rate ratio 9.375 ; 95\% CI: 8.998 to 9.768 ).

In this study, the annual incidence of UEDVT was 0.098 per 1,000 inhabitants and these thromboses accounted for $14 \%$ of the total number of proximal deep vein thromboses of the extremities. The two leading risk factors for UEDVT were the presence of a central venous catheter and active cancer. UEDVT was associated with pulmonary embolism in $8.3 \%$ of the cases.

Herein we provide for the first time a direct estimate of the incidence of UEDVT in an unselected population of patients diagnosed in hospitals and the community. Previously there have been only indirect estimates of the incidence of UEDVT, with annual rates varying from 0.04 to 0.1 per 1,000 inhabitants. ${ }^{3}$ In the only available population-based study, the incidence of UEDVT was 0.036 per 1,$000 ;^{9}$ however, not every case of UEDVT that occurred in the studied population was recorded explaining in part the difference observed with our data. As initially hypothesized, the incidence we found was within the former upper estimates and is probably the result of the increased use of central venous catheters to deliver chemotherapy, drugs, and parenteral nutrition. The use of peripherally inserted central catheters has increased substantially in recent years - not only in cancer patients - because they are easy to insert and remove at the bedside; however, these catheters are associated with an even higher risk of deep vein thrombosis than centrally placed catheters. ${ }^{10}$

Notably, the incidence of primary UEDVT remained stable across age categories, whereas the incidence of secondary UEDVT increased with increasing age. Primary UEDVT is a rare disorder with a previously estimated annual incidence of 0.02 per 1,000 inhabitants. ${ }^{11}$ Our data suggest a lower incidence rate and emphasize the fact that primary UEDVT can happen at any age.
Nevertheless, young patients with UEDVT are more likely to present with primary thrombosis than older patients, the latter being those who more often need a central venous catheter or who have active cancer.

Our observation of an $8.3 \%$ frequency of symptomatic pulmonary embolism associated with UEDVT is also in line with prior reports. ${ }^{3}$ This low frequency, when compared to that in patients with lower-extremity deep vein thrombosis, could mask higher rates of silent pulmonary embolism. In fact, when systematic lung imaging is performed in patients without symptoms of pulmonary embolism, the prevalence of asymptomatic pulmonary emboli can reach $36 \%{ }^{12}$ Small pulmonary emboli, secondary to a low load of thrombotic material in the upper extremities, may explain this high rate of silent events.

The decision to exclude asymptomatic line-associated UEDVT diagnosed in the workup of pulmonary embolism, or UEDVT revealed by dysfunction of a central line from our study led to an underestimation of the incidence of clinically significant UEDVT. However, in order to compare our data with those of previous studies, it was necessary to use a common definition of symptomatic UEDVT.

We acknowledge that the sample size is also a limitation of our study leading to wide confidence intervals. However, we performed a prospective study of all symptomatic proximal UEDVT cases diagnosed in hospitals or in the community in a well-defined geographic area whose natural geographic limits ensure exhaustive records and accuracy of estimates. ${ }^{7}$ Furthermore, we reviewed and adjudicated all patients' charts in order to confirm and validate the diagnosis of UEDVT and we chose a 2-year inclusion period to increase the number of observed cases and avoid the effect of any seasonal variation over a shorter period of observation.

In conclusion, UEDVT is an infrequent manifestation of venous thromboembolism that can be associated with symptomatic pulmonary embolism. The risk factors for UEDVT are easily identifiable; however, how to improve the prevention of these events needs further investigation.

Aurélien Delluc, ${ }^{1,2}$ Raphaël Le Mao, ${ }^{1,3}$ Cécile Tromeur, ${ }^{1,3}$ Nathalie Chambry, ${ }^{1}$ Hélène Rault-Nagel, ${ }^{1}$ Luc Bressollette, ${ }^{1,3}$ Dominique Mottier, ${ }^{1,4}$ Francis Couturaud $d^{1,3,4}$ and Karine Lacut ${ }^{1,3}$

${ }^{1}$ EA 3878 (GETBO), Brest University, France; ${ }^{2}$ Division of Clinical Hematology, the Ottawa Hospital; Clinical Epidemiology Program, Ottawa Hospital Research Institute, ON, Canada; ${ }^{3}$ Department of 
Vascular Medicine, Internal Medicine, and Chest Diseases, Hôpital de la Cavale Blanche, 29609 Brest Cedex, France and ${ }^{4}$ INSERM CIC 1412, Hôpital de la Cavale Blanche, 29609 Brest Cedex, France

Correspondence: adelluc@toh.ca doi:10.3324/haematol.2018.194951

Information on authorship, contributions, and financial \& other disclosures was provided by the authors and is available with the online version of this article at www. haematologica.org.

\section{References}

1. Kearon C, Akl EA, Comerota AJ, et al. Antithrombotic therapy for VTE disease: Antithrombotic Therapy and Prevention of Thrombosis, 9th ed: American College of Chest Physicians Evidence-Based Clinical Practice Guidelines. Chest. 2012;141(2 Suppl):e419S-494S

2. Flinterman LE, Van Der Meer FJM, Rosendaal FR, Doggen CJM. Current perspective of venous thrombosis in the upper extremity. J Thromb Haemost. 2008;6(8):1262-1266.

3. Grant JD, Stevens SM, Woller SC, et al. Diagnosis and management of upper extremity deep-vein thrombosis in adults. Thromb Haemost. 2012;108(6):1097-1108.

4. Joffe HV, Goldhaber SZ. Upper-extremity deep vein thrombosis. Circulation. 2002;106(14):1874-1880.

5. Joffe HV, Kucher N, Tapson VF, Goldhaber SZ. Upper-extremity deep vein thrombosis: a prospective registry of 592 patients. Circulation. 2004;110(12):1605-1611.

6. Muñoz FJ, Mismetti P, Poggio R, et al. Clinical outcome of patients with upper-extremity deep vein thrombosis: results from the RIETE Registry. Chest. 2008;133(1):143-148.

7. Delluc A, Tromeur C, Le Ven F, et al. Current incidence of venous thromboembolism and comparison with 1998: a community-based study in Western France. Thromb Haemost. 2016;116(5):967-974

8. http://www.insee.fr/fr/ themes/tableau_local.asp?ref_id=POP1B \&millesime $=2011 \&$ niveau $=1 \&$ typgeo $=A R R \&$ codgeo $=291$.

9. Isma N, Svensson PJ, Gottsäter A, Lindblad B. Upper extremity deep venous thrombosis in the population-based Malmö thrombophilia study (MATS). Epidemiology, risk factors, recurrence risk, and mortality. Thromb Res. 2010;125(6):e335-338.

10. Geerts W. Central venous catheter-related thrombosis. Hematology Am Soc Hematol Educ Program. 2014;2014(1):306-311

11. Lindblad B, Tengborn L, Bergqvist D. Deep vein thrombosis of the axillary-subclavian veins: epidemiologic data, effects of different types of treatment and late sequelae. Eur J Vasc Surg. 1988;2(3):161165.

12. Prandoni P, Polistena P, Bernardi E, et al. Upper-extremity deep vein thrombosis. Risk factors, diagnosis, and complications. Arch Intern Med. 1997;157(1):57-62. 